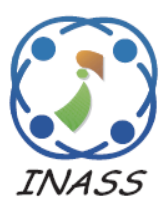

http://www.inass.org/

\title{
Utility-Based Horizontal Handover Decision Method for Vehicle-to-Vehicle Communication in VANET
}

\author{
Sahirul Alam ${ }^{1}$ \\ Selo Sulistyo ${ }^{1 *}$ \\ I Wayan Mustika ${ }^{1}$ \\ Ronald Adrian ${ }^{1}$ \\ ${ }^{I}$ Department of Electrical Engineering and Information Technology, \\ Universitas Gadjah Mada, Indonesia \\ * Corresponding author’s Email: selo@ugm.ac.id
}

\begin{abstract}
Vehicular ad hoc network (VANET) is the emerging wireless communication technology in vehicular domain to support the safety and non-safety purposes in transportation. Despite the potential implementation of VANET, some challenges mainly due to the dynamic environment need to be addressed and tackled. In this paper, the horizontal handover especially in vehicle-to-vehicle (V2V) connection is investigated. The dominant handover decision method in wireless network, namely received signal strength (RSS) based method, is actually inappropriate for the implementation in V2V VANET. Therefore, a utility based handover decision method is proposed in this paper. The utility function is formulated based on the value of signal to noise ratio (SNR) and the load of network. Despite the fluctuation of SNR, the proposed method can make the better handover decision i.e. by reducing the ping pong effect and improving the throughput by selecting the network with lower load. In this research, the proposed method is evaluated through simulations. The results of simulations show that the proposed method can improve the performance of $\mathrm{V} 2 \mathrm{~V}$ handover in terms of throughput and handover rate compared with the RSS based method.
\end{abstract}

Keywords: Utility, Handover decision, Vehicle-to-vehicle communication, Vehicular ad hoc network.

\section{Introduction}

The growing number of vehicles and the needs of convenience and safety in transportation have spurred the advance of intelligent transportation technology. Another motivation is to solve the traffic problems such as congestion and the optimal route suggestion or the driving assistance. To support those purposes, the vehicles need a network system to enable communication between vehicle to infrastructure (V2I) and vehicle to vehicle (V2V). Thus, the vehicular ad hoc network (VANET) is the emerging technology to actualize those purposes [1]. To enable communication between vehicles, a device, usually called on board unit (OBU), is installed on each vehicle. According to the Federal Communications Commission (FCC), the allocated spectrum for VANET is $5.85-5.925 \mathrm{GHz}$ with a 75 $\mathrm{MHz}$ bandwidth. While the standard of communication released by IEEE is the $802.11 \mathrm{p}$ [2].
In spite of its potential applications, VANET has several challenges that draw the interest of any communities related with the field, such as government, industry, and researchers. One of the major technical challenges in VANET is the highly dynamic environment [3]. The mobility of vehicles, as well as the density of vehicles, could have the high variation. Hence, it is not easy to maintain the stability and the quality of the communication links. The conventional network topologies are regarded as not appropriate for the implementation in VANET. The hierarchical network topology known as clustering is more considered for VANET [4]. In clustering, several vehicles with the same characteristics are grouped into a cluster. The characteristics could be the geographical position, vehicle speed and direction, and so on. Thus, clustering is expected to enable the establishment of more stable $\mathrm{V} 2 \mathrm{~V}$ communications. A cluster consists of a cluster head which acts as the central of cluster and responsible for the cluster management. 
The other vehicles except the cluster head are called as the cluster members.

As the vehicles have the high mobility, the change of communication link is inevitable. The change of communication link is known as handover. In cellular network, when a mobile user moves outside the coverage area of the previous cell, the mobile user will perform handover or change the connection to the cell that currently covers the area. In VANET environment, the handover could be performed by a vehicle that moves from the coverage of a road side unit (RSU) to the coverage of another RSU. This type of handover is called as horizontal handover [5], i.e. the change of communication link within a same technology. Another type of handover is the vertical handover, i.e. the change of connection between two technologies. For example, a mobile user changes connection from cellular to $\mathrm{WiFi}$, and vice versa.

This paper studies the horizontal handover. However, it is not the handover from an RSU to another RSU (V2I handover), but the handover from a cluster to another cluster (V2V handover) as illustrated in Fig. 1. The reason is that the handover between two RSUs is simpler and can be handled by the existing methods. It is because the RSUs are installed on a static place, thus the trend of the received signal strength (RSS) can be predicted for the purpose of handover decision. Meanwhile, the handover between clusters is basically the change of communication link between vehicle nodes, since the cluster head is also a vehicle. The problem becomes more complicated since all the involved nodes are moving. In this case, the RSS fluctuates over time, and the proper handover decision becomes more difficult. In this paper, a utility is proposed for handover between clusters in VANET. The utility is formulated based on the signal to noise ratio (SNR) and the number of cluster members. The main contributions of this paper and the advantage of the proposed method are as follow.

1. The investigation of handover problem in $\mathrm{V} 2 \mathrm{~V}$ VANET especially due to the high fluctuation of RSS as the result of the dynamic mobility of vehicles and the dynamic environment.

2. The proposed utility for handover decision method utilizes the information about SNR and network load which can be obtained by each vehicle. Thus, the proposed handover decision method can be performed in distributed manner, which is preferable in VANET to reduce overhead.

3. The proposed handover decision method can provide the better result in terms of throughput and handover rate compared to the dominant method for handover decision in wireless networks.

The rest of this paper is organized as follows. The brief review of some related works and the difference with this research are presented in section 2. The proposed handover decision method and the formulation of the utility are described in section 3 . The description about how the simulations were conducted, the results of simulations, and the analysis of simulation results are presented in section 4. Finally, the conclusion of this paper is given in section 5 .

\section{Related works}

The study of handover in VANET has been gaining the interest as several articles can be found in literature. However, those studies mainly about the V2I handover between RSUs or access points (APs) such as in [6-9]. The other studies investigate the vertical handover, i.e. the handover between RSU, AP, and cellular base station (BS), such as in [10-12]. There is a significant difference between V2I handover and V2V handover especially in the fluctuation of RSS. Due to path loss, the RSS will decrease or increase proportional to the distance between transmitter and receiver and because of shadowing, the RSS will fluctuate over time. The instantaneous value of RSS can increase or decrease randomly because of shadowing effect as depicted in Fig. 2. However, since the infrastructures (e.g. RSU, AP, and cellular BS) are placed in static place, in spite of the fluctuation due to shadowing, the trend of RSS value can still be predicted. Meanwhile in $\mathrm{V} 2 \mathrm{~V}$ connection, both the nodes are mobile. Hence, the trend of RSS value is hard to predict, mainly because of the mobility of vehicle nodes. It is as hard as predicting the mobility of vehicle which depends on the driver's preference. For example, Fig. 3 shows the plot of RSS from two cluster heads toward a vehicle. In this example, the vehicle moves at a distance from cluster head 1 , but the speed difference between the vehicle and cluster head is not so high. Thus, the vehicle moves along with the cluster head 1 as if the vehicle follows the

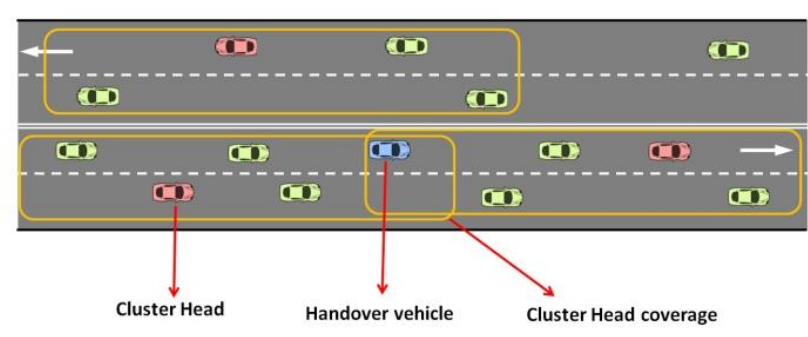

Figure.1 Cluster to cluster (V2V) handover 


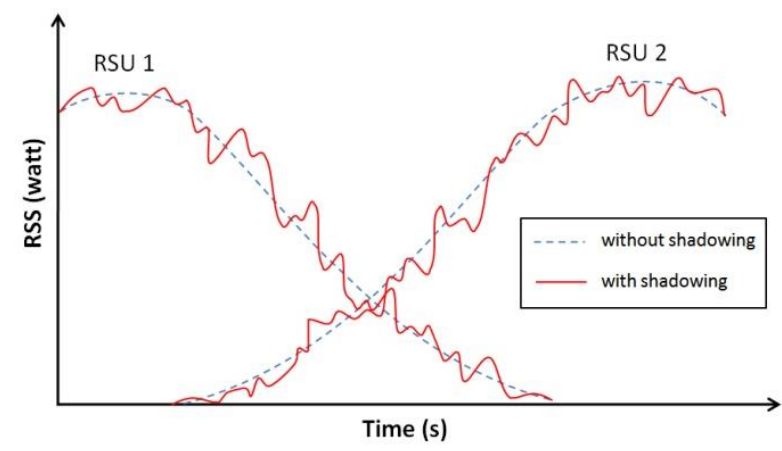

Figure.2 RSS fluctuation in V2I connection

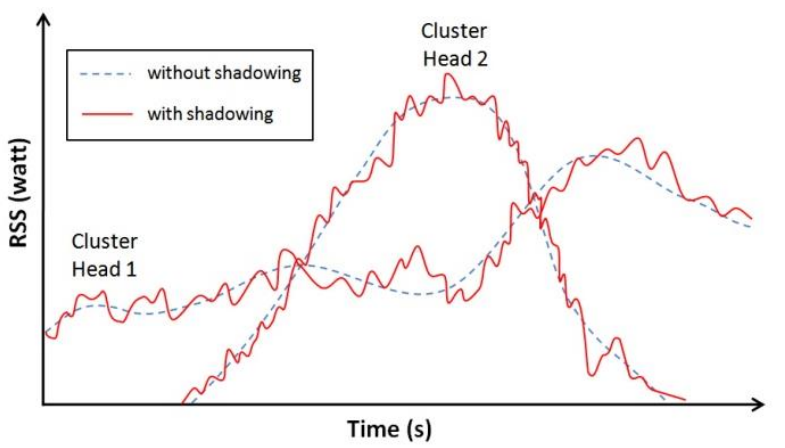

Figure.3 RSS fluctuation in V2V connection

cluster head. Therefore, the RSS fluctuates proportional to the change of distance in addition to the shadowing effect. During the movement, the vehicle approaches cluster head 2 which has the lower speed. Since the speed difference is relatively high, the vehicle can overtake cluster head 2 . In this case, the vehicle moves as if it passes by the RSU. Hence, the fluctuation of RSS resembles to the fluctuation of RSS in V2I connection. This example is just a piece of condition in highly dynamic environment of VANET. Many other conditions could happen, and thus the handover decision needs more reliable method in order to improve the quality of communication in V2V VANET.

The study of V2V handover in VANET is presented in [13]. The system model in [13] assumed a vehicle node performing handover between relay vehicles (RVs). The concept of relay vehicle resembles the cluster head in clustering concept, where the RV has the responsibility to relay the messages and manage the $\mathrm{V} 2 \mathrm{~V}$ communication. However, RV is limited to the large vehicle such as bus. Thus, it has a slightly different concept compared to clustering, where the cluster head is selected based on certain criteria such as the position (at the center of cluster geographical position) or the smallest speed difference with the average speed of cluster members. Moreover, the study of handover in [13] dealt with the handover algorithm or the procedure of performing handover.
That is by utilizing the vehicles from the opposite direction to provide the information about e.g. the available channel at the front RV. Thus it can reduce the handover latency. Whereas, the handover study in this paper focuses on the handover decision i.e. when the handover should be performed and to which vehicle node (cluster head) the handover should be performed. The handover decision is also an essential factor that affects the stability and the quality of communications. Handover should be decided wisely so that the ping pong effect can be avoided and the new established connection after handover can give the benefits instead of detriments.

The studies about handover decision in VANET are not as many as the handover decision in the cellular or wireless networks in general. Nevertheless, it can be noted that the prominent methods in handover decision (especially in horizontal handover) use RSS as the prime factor [5,14-15]. Therefore, the utility proposed in this paper also utilizes the RSS as one of the factors for handover decision. To solve handover problem in VANET, especially in V2V connection, RSS alone is not sufficient. The conventional RSS-based handover decision methods are straightforward and hence, they cannot handle VANET condition that is very dynamic. As the result, the conventional methods are prone to higher handover rate. Furthermore, higher handover rate and topology change cause the frequent change to the network load. This later affects the throughput of communication. Therefore, more factors beside RSS should be considered in V2V handover decision.

Other utility-based methods for handover decision in wireless networks utilize particular factors such as monetary or cost of service [16]; signal to interference plus noise ratio [17]; throughput and load of cell [18]; cost of service, security, power requirements, user preference, quality of service, and velocity [15]. However, several factors above are not relevant in VANET environment such as the cost of service, since the $\mathrm{V} 2 \mathrm{~V}$ communication in VANET is not charged for the service unlike the cellular service. Power conservation is also irrelevant since the power of OBU is supplied by the battery of vehicle. Security and signal interference are crucial, however they are hard to approximate [5], especially in vehicular networking environment. Moreover, the estimation of those parameters will result more overhead or latency in handover process. Therefore, the proposed utility in this paper is formulated using RSS and network load (i.e. the number of cluster members), since those parameters can be easily 
approximated. Besides that, only small computation load is needed.

\section{Proposed handover decision method}

RSS which is also known as the reference signal received power (RSRP) determines the SNR as well as the throughput of a communication link. Thus, if a vehicle node is located at an overlapping coverage area of two cluster heads, the vehicle node will select the cluster head that transmits the higher RSS. Here, if the cluster head currently connected with the vehicle node has lower RSS than the other cluster head, according to the RSS-based handover, the vehicle node will perform handover and change the connection with the cluster head which transmits the higher RSS. This handover decision is subject to the following equation.

$$
Q_{i}>Q_{s}+H y s_{S}
$$

where $Q_{i}$ is the RSS from the neighbor cluster head, $Q_{S}$ is the RSS from currently serving cluster head, and $\mathrm{Hys}_{S}$ is the hysteresis or the margin of RSS difference between serving cluster head and neighbor cluster head.

One of the purposes of using RSS as the parameter for handover decision is to obtain the higher SNR and throughput. Theoretically, the higher SNR will result the higher throughput. However, the throughput is limited by the maximum data rate of the system. While the SNR is lower, the communication can still be conducted, but the throughput obtained is also lower. The amount of throughput is determined by the packet delivery ratio (PDR). The value of PDR ranges from 0 to 1 , where the PDR $=1$ is regarded as the high quality transmission, since all the packets are delivered successfully. According to the research in [9], PDR $(\rho)$ of IEEE 802.11p transmission which is modeled using [19], can be formulated as follows.

$$
\rho=[1-1.5 \operatorname{erfc}(0.45 \sqrt{\Gamma})]^{L}
$$

where $\Gamma$ is the SNR in linear (not in $\mathrm{dB}$ ) and $L$ is the length of packet. The example of PDR plot using packet length 1024 is depicted by the Fig. 4 .

In Fig. 4 , the PDR = 1 can be achieved when the SNR value is above $30 \mathrm{~dB}$. Furthermore, since the maximum PDR can be achieved at $30 \mathrm{~dB}$ of SNR, the higher value of SNR will not result the higher PDR. In spite of this, the higher SNR is still preferred to anticipate the fluctuation of SNR so that the higher PDR can be maintained. In the highly

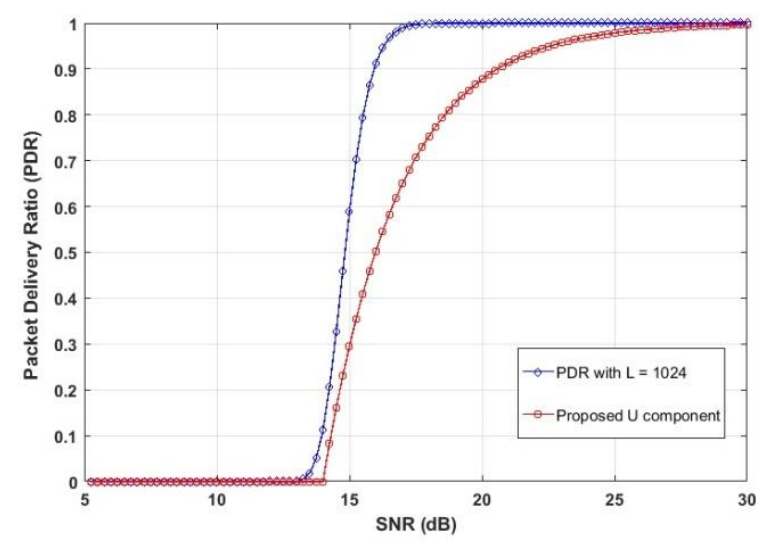

Figure.4 PDR versus SNR and the proposed utility component

dynamic environment of VANET, the value of SNR will fluctuate especially due to the mobility of vehicles. A vehicle can overtake the cluster head, moves along with the cluster head, or left behind by the cluster head. Hence, the trend of SNR value is difficult to predict. The decreasing value of SNR could rise again and vice versa. This circumstance could lead the ping pong effect in handover, i.e. a vehicle performs handover to a new cluster head, but a few moments later reverts the connection to the previous cluster head. The ping pong effect is very detrimental in communication process, especially for the routing protocol.

The proposed utility for handover decision aims to obtain the higher throughput and reduce the ping pong effect. The parameters used in the calculation of utility are the SNR and the number of cluster members. The utility of connection with cluster head $i\left(U_{i}\right)$ is formulated as follows.

$$
U_{i}\left(\Gamma_{i}, M_{i}\right)= \begin{cases}\frac{1-e^{-\lambda\left(\Gamma_{i}-\Gamma_{\text {thd }}\right)}}{M_{i}} & \Gamma_{i} \geq \Gamma_{\text {thd }} \\ 0 & \Gamma_{i}<\Gamma_{\text {thd }}\end{cases}
$$

where $\Gamma_{i}$ is the SNR of connection with cluster head $i, \Gamma_{\text {thd }}$ is the threshold value of SNR, $M_{i}$ is the number of cluster members in cluster $i$ including the handover vehicle, and $\lambda$ is the exponential coefficient. From Eq. (3), the utility will have maximum value if there is only one member in the cluster. Meanwhile, the value of nominator in Eq. (3) is determined by $\Gamma_{i}$, since $\lambda$ and $\Gamma_{\text {thd }}$ is the predefined parameters. The plot of utility function using $\lambda=0.35, \Gamma_{\text {thd }}=14 \mathrm{~dB}$ and $M=1$ is shown in Fig. 4. It can be seen that the value of utility is related with PDR. However, for the same value of PDR and utility, the utility demands the higher SNR value. This is intended to give the margin so that the higher PDR can be achieved. It can be noted that at 
certain value of SNR, the utility value is saturated. Hence, the fluctuation of SNR above this value will not affect the utility. This is intended to attain more stable connection and reduce the ping pong effect. Moreover, it is fine since at that SNR value, the PDR is equal 1 which is the best condition for transmission with high delivery rate. Thus, when the SNR value has been able to provide maximum PDR, the handover decision will consider the lower network load so that the channel resource utilization can be optimized and the higher throughput can be obtained. Furthermore, the utility curve can be adjusted to conform to the system requirement, i.e. by changing the value of $\lambda$ and $\Gamma_{t h d}$. For example, by setting the higher value of $\Gamma_{t h d}$, the curve will move to the right side, and thus the higher value of SNR is required to achieve certain utility value. Then, to make the curve become more sloping, the value of $\lambda$ can be decreased, and thus the higher SNR is required to achieve the utility $=1$ as shown in Fig 5 .

The mechanism of the proposed handover decision method is as follows. The handover decision is performed in distributed manner. However, the vehicle performing handover decision (vHO) needs the information about SNR of nearby cluster heads and the number of members in those clusters. The value of SNR can be measured by the vehicle's OBU. Meanwhile the information about the number of cluster members can be obtained from the cluster gateway, especially if the front cluster head is still too far and hence cannot send the information properly to the vehicle. Moreover, it is recommended that the information about number of cluster members is provided by the cluster gateway, thus the task of cluster head can be reduced. Cluster gateway is basically a member of cluster which the position is at the edge of cluster head coverage as illustrated in Fig. 6. The method to select a gateway node is presented in [20]. Cluster gateway (GW) is

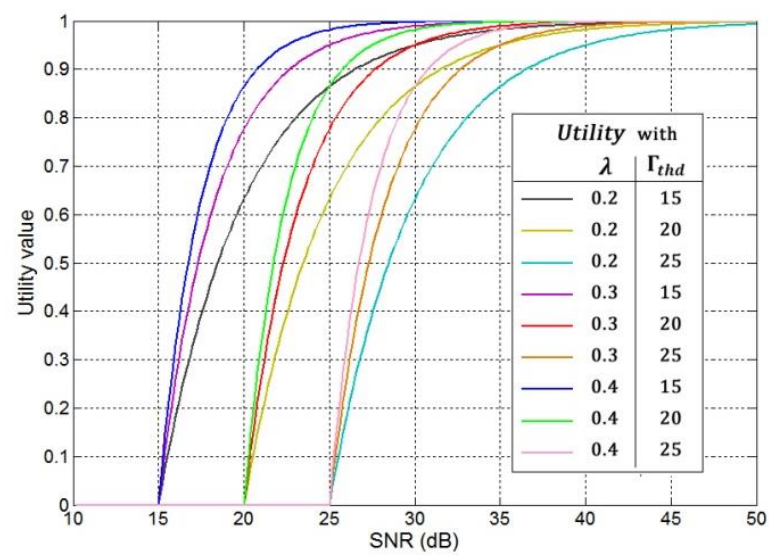

Figure.5 Plot of utility value with different parameters setting

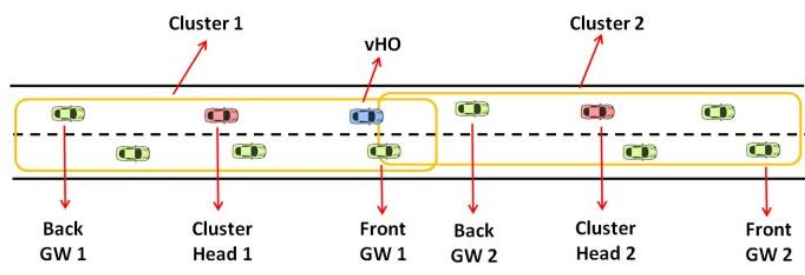

Figure.6 Cluster gateway in cluster configuration

initially designated to relay the messages to another cluster. However, the role of cluster gateway can be extended to aid the handover process.

In utility-based handover, a vehicle performs handover if there is a cluster head with the higher utility value than the cluster head currently connected. If there are two or more cluster heads with the higher utility value, then the cluster head with the highest utility will be selected. The proposed handover decision procedure is presented in algorithm 1. Since the proposed method is performed in distributed manner, the algorithm 1 uses the perspective of $\mathrm{vHO}$. The handover decision is evaluated at every beacon interval $(B I)$. Even though $B I$ is user defined, its value affects the system performance as follows. If $B I$ is short, then the handover decision can be evaluated more often. Hence, it can avoid late handover where the vehicle loses a connection with current cluster, but not yet connects with the new cluster. However, the short $B I$ will increase the data traffic and may cause the transmission overhead. During handover decision process, vHO gathers the information about SNR and number of members at any nearby clusters $\left(N_{c}\right)$. Afterwards, the utility value of each nearby cluster $\left(U_{i}\right)$ is calculated, and the cluster with the highest utility $\left(C_{\text {best }}\right)$ is found. If $C_{\text {best }}$ is different with the cluster currently serving $\left(C_{s}\right)$, then vHO will perform handover from $C_{s}$ to $C_{\text {best }}$.

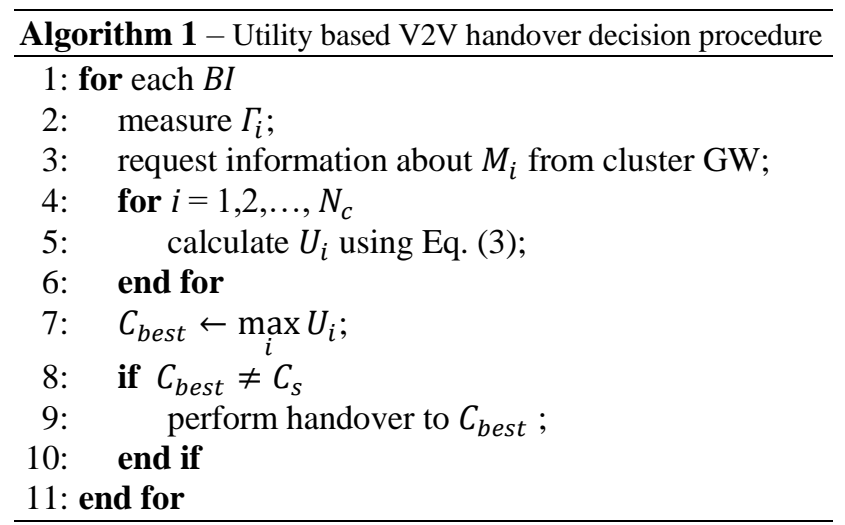




\section{Simulation results and discussion}

\subsection{Simulation setup}

In this research, simulations were performed using a vehicle mobility simulator namely simulation of urban mobility (SUMO)[21] and MATLAB for simulating the handover in VANET. The details of the system model built for simulation are as follows. In SUMO, a road network with two opposite directions was created. The road's length is 2 kilometers and there is a road junction in the middle of the road network as shown in Fig. 7(a). The road has three lanes in each direction as shown in Fig. 7(b).

There are 4 types of vehicles deployed in this simulation. Each type of vehicle has different characteristics in terms of average speed and dimension or size of vehicle. Cars are the smallest vehicle represented using yellow color in SUMO graphic user interface (GUI) and they have average speed $18 \mathrm{~m} / \mathrm{s}$. Trucks have bigger size than car, represented using green color and they have average speed $14 \mathrm{~m} / \mathrm{s}$. Coaches are the largest vehicle, represented using orange color, and they have average speed $12 \mathrm{~m} / \mathrm{s}$. The last type of vehicle, called by handover vehicle (vHO) is basically a car and represented using blue color. During simulation, vHO was observed. How many times vHO performed handover and how the throughput was obtained during simulation became the main focuses of observation. Furthermore, to evaluate the performance of the proposed handover decision method, the simulations were repeated by altering the average speed of $v H O$ from $17-25 \mathrm{~m} / \mathrm{s}$ with the increment $2 \mathrm{~m} / \mathrm{s}$. The vehicles could enter or leave the road network from the border of the roads (right side or left side in Fig. 7(a)) and the border of the roads connected with the road junction. The

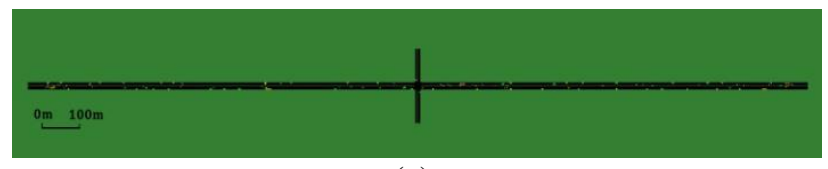

(a)

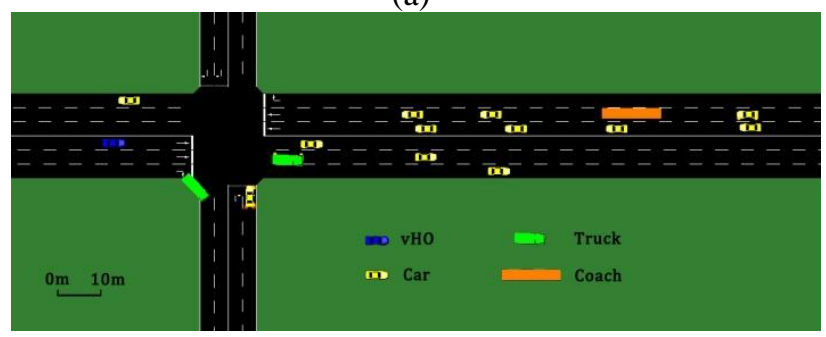

(b)

Figure.7 SUMO road network for handover simulation: (a) road junction and (b) three lanes in each direction environment of simulation was assumed in the highway road, and thus the number of vehicles in the road network at a time could be varied from 100 to 150 .

The results of simulation in SUMO were the position of vehicles (in $x-y$ coordinate) and the speed of vehicles. These results were used for handover simulation in MATLAB afterwards. The clustering method used in this research is based on [20], since it is considered as the stable clustering method and it has just proposed recently. Moreover, it is not difficult to implement the clustering method using MATLAB programming. As the clusters were formed, the $\mathrm{vHO}$ was deployed form the left border of the road network and it attempted to establish V2V connection with any nearby cluster heads based on the handover method assumed. However, both the clustering method and the vHO are restricted to establish connection with the vehicles from opposite direction. The reason is that the lifetime of the established connection will be short and it is not desired by the most of clustering method nowadays.

The simulations were conducted using 5 different settings of the vHO average speed. Thus, there are five data sets of vehicle position generated from SUMO. Then, each data set was used for handover simulation in MATLAB. For each data set, handover is simulated using the proposed utilitybased method and RSS-based method for comparison. Since the simulations assumed highway environment, the RSS $(Q)$ was calculated using the following formula.

$$
Q=p 10^{\left(P L_{d B} / 10\right)}
$$

where $p$ is the power of transmission and $P L_{d B}$ is the path loss in decibel. $P L_{d B}$ in highway environment is calculated using the following formula [22].

$$
P L_{d B}=-\left(54.02+16.6 \log _{10} d\right)+S_{\sigma}
$$

where $d$ is the distance from the cluster head to the vHO and $S_{\sigma}$ denotes the shadowing with standard deviation $3.68 \mathrm{~dB}$. The SNR of V2V connection is calculated based on the value of RSS using the following formula.

$$
\Gamma_{d B}=10 \log _{10}\left(\frac{Q}{B N_{0}}\right)
$$

where $\Gamma_{d B}$ denotes SNR in decibel, $B$ is the channel bandwidth, and $N_{0}$ is the white noise intensity. 
In this research, in addition to the SNR, there are two metrics for performance evaluation of the proposed handover decision method, i.e. throughput and handover rate. Throughput indicates the amount of successful data transmission per unit of time. In this research, throughput $(H)$ is calculated as follows.

$$
H=\rho R_{b} T_{e}
$$

where $\rho$ is the PDR which is calculated using Eq. (2), $R_{b}$ is the channel bit rate, and $T_{e}$ denotes the expected number of time slot occupied per second. The value of $T_{e}$ is inversely proportional with the number of cluster member. The maximum value of $T_{e}$ is obtained if the cluster only has one member, and thus the whole time slot is used by that cluster member. On the contrary, the higher number of cluster member, the lower value of $T_{e}$ and hence the throughput decreases significantly. The handover rate denotes the rate of handover per unit of time. It can be calculated based on the number of handover occurrences divided by the duration of simulation. The duration of simulation is determined by the average speed of vHO. The faster $\mathrm{vHO}$, the shorter duration of simulation, as vHO can reach the end of the road network faster.

For evaluating the performance of the proposed method, handover decision simulation using RSSbased and time average of RSS (TA-RSS)[23] methods are performed. Furthermore, the values of parameters used in these simulations are listed in Table 1.

\subsection{Results and analysis}

The results of handover simulations as described in simulation setup are presented below. Fig. 8 shows the average SNR value of vHO during simulation. It can be seen that RSS-based and TA-RSS methods have slightly higher SNR average than the proposed utility based handover. This is because RSS-based and TA-RSS methods always select the

Table 1. Parameters value

\begin{tabular}{|l|l|}
\hline \multicolumn{1}{|c|}{ Parameter } & \multicolumn{1}{c|}{ Value } \\
\hline Transmission power $(p)$ & $20 \mathrm{dBm}$ \\
\hline Channel bandwidth $(B)$ & $10 \mathrm{MHz}$ \\
\hline White noise intensity $\left(N_{0}\right)$ & $-174 \mathrm{dBM} / \mathrm{Hz}$ \\
\hline SNR threshold $\left(\Gamma_{\text {thd }}\right)$ & $14 \mathrm{~dB}$ \\
\hline Exponential coefficient $(\lambda)$ & 0.35 \\
\hline RSS hysteresis $\left(H_{y}\right)$ & $10^{-9} \mathrm{watt}$ \\
\hline Channel bit rate $\left(R_{b}\right)$ & $12 \mathrm{Mbps}$ \\
\hline Packet length $(L)$ & 1024 bytes \\
\hline Beacon interval $(B I)$ & $0.5 \mathrm{~s}$ \\
\hline Cluster head range & $300 \mathrm{~m}$ \\
\hline
\end{tabular}

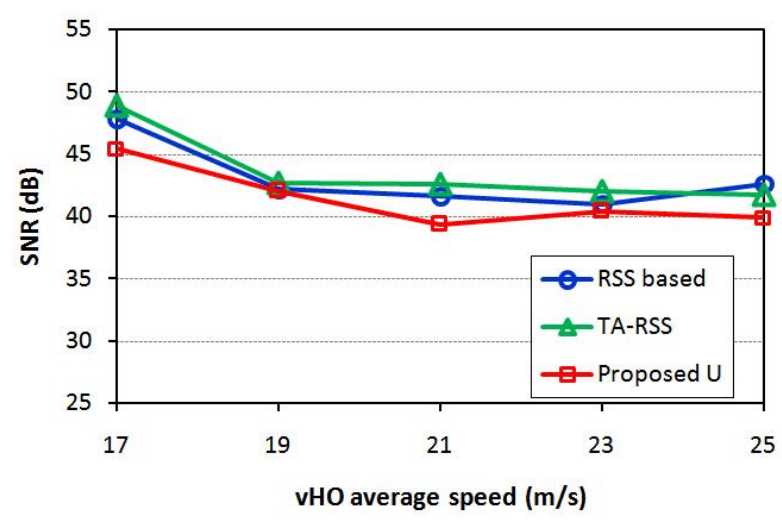

Figure.8 SNR average of vHO

cluster head with the highest RSS. Meanwhile, the proposed method does not always select cluster head with the highest RSS, but based on the highest utility value. The utility function consists of two inputs namely SNR and the number of cluster members.

Although RSS-based and TA-RSS methods have the higher average of SNR than the proposed method, the throughput obtained by RSS-based and TA-RSS methods do not follow this result accordingly. On the contrary, the proposed method can obtain the higher average of throughput than RSS method as shown in Fig. 9. This is because when RSS-based and TA-RSS methods select the cluster head with the highest RSS , the network load at that cluster head is probably high i.e. depends on the number of cluster members. When the network load is high, then the data traffic congestion is also high and the portion of time slots occupied by $\mathrm{vHO}$ becomes less. Consequently, the obtained throughput decreases significantly. Meanwhile, the proposed method also considers the number of cluster members in the calculation of utility function. Hence, in the proposed method, the highest RSS cluster head will not always be selected for handover especially if the load at that cluster head is high. But, the cluster head with sufficient RSS and low network load is preferable. Therefore, this strategy can result the higher throughput average for vHO.

In term of handover rate, the proposed method has the better result than RSS-based and TA-RSS handover as depicted in Fig. 10. In a highly dynamic environment such as VANET, RSS based handover can be at a disadvantage. The mobility of vehicles can cause the fluctuation of RSS in V2V connection. Consequently, the frequent handover and the ping pong effect occur as the result of this condition. TARSS can achieve lower handover rate than RSSbased handover decision due to time averaging 


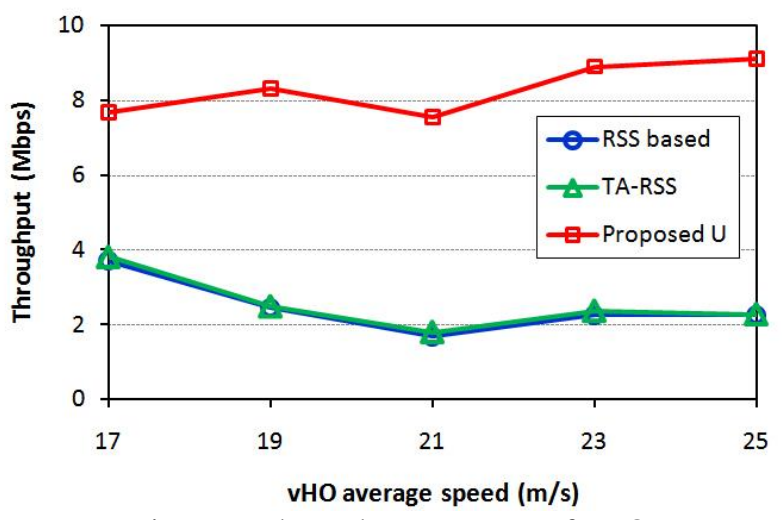

Figure.9 Throughput average of vHO

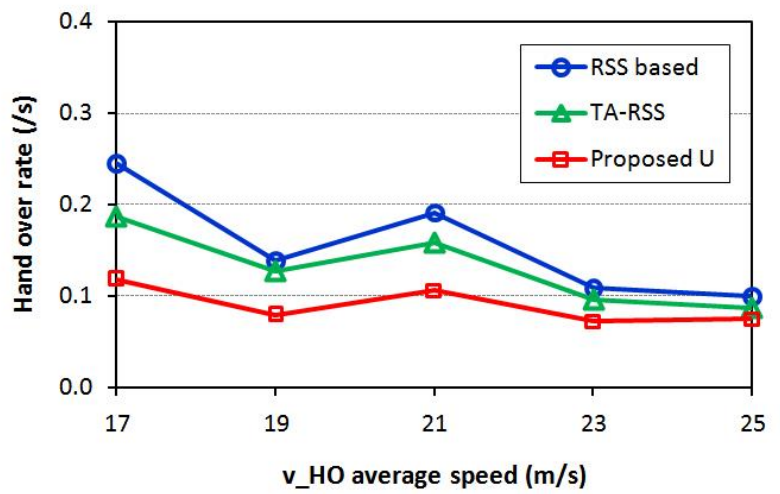

Figure.10 Handover rate of $\mathrm{vHO}$

method. By time averaging the RSS of nearby cluster head, vHO can select the more promising cluster head for the longer term. However, handover rate reduction is less significant. This is because TA-RSS uses RSS threshold to trigger handover which is ineffective for the implementation in $\mathrm{V} 2 \mathrm{~V}$ connection with high RSS fluctuation.

The proposed method has lower handover rate compared to RSS-based and TA-RSS handover decision. This is because in utility function, the value of SNR has a point where it gives the maximum contribution to the utility value as in Fig. 4. For example, if a $40 \mathrm{~dB}$ SNR gives the maximum contribution to the utility value, then the fluctuation of SNR above $40 \mathrm{~dB}$ will result no change in the value of utility. In this case, the variable that can change the value of utility is the number of cluster members. Therefore, as long as the fluctuation of SNR does not change the value of utility and the current cluster head has lower network load than other nearby cluster heads, vHO will not perform handover.

The performance of handover decision methods related with the average of $\mathrm{vHO}$ speed can be analyzed but cannot be confirmed. It is because the handover performance is affected by the mobility of the other vehicles. However, the following is the intuitive analysis of the simulation results. At the lower speed, e.g. $17 \mathrm{~m} / \mathrm{s}$, vHO may have the lower average speed than the cluster head. Thus, vHO can be left behind by a cluster head and another cluster head can catch up from behind. In this condition, RSS fluctuation is high and hence the RSS based handover suffers ping pong effect even though the average SNR is high. In this condition, the proposed method also has a slightly higher handover rate than in the other conditions. It indicates that at this speed, the network condition is very dynamic. When the average speed of $\mathrm{vHO}$ is $19 \mathrm{~m} / \mathrm{s}$, the network condition is not as dynamic as the previously mentioned condition. This is indicated with the lower handover rate than in previous condition. At this condition, vHO average speed is almost same with the average speed of the cluster head. Thus, vHO moves along with the cluster head and as the result, the connection can be maintained for the longer duration. When the average speed of vHO is $21 \mathrm{~m} / \mathrm{s}$, vHO moves slightly faster than the cluster head and thus it can leave a cluster head and catch up the cluster head in front. However, since the vHO is not significantly faster than the cluster heads, the network condition is also dynamic as in the condition when vHO average speed is $17 \mathrm{~m} / \mathrm{s}$. When vHO moves significantly faster than the cluster head, it can be considered as if the vHO passes by the static nodes. Therefore, the characteristics of this condition are almost same with the handover between RSUs. In this condition, RSS value has a trend i.e. the RSS of current cluster head gradually decreases and the RSS of the cluster head in front gradually increases. Therefore, RSS based handover performance becomes better at this condition.

\section{Conclusion}

In vehicular networking, the network condition is very dynamic. Hence the RSS trend, especially in $\mathrm{V} 2 \mathrm{~V}$ connection, is very hard to be predicted. Therefore, the dominant handover method in wireless network is not appropriate to be implemented in V2V connection. In this paper, a utility is proposed for handover decision in $\mathrm{V} 2 \mathrm{~V}$ VANET. The utility is formulated based on the SNR value and the load of network, i.e. the number of cluster members. The SNR value is needed to estimate the PDR, thus as long as the sufficient PDR value is obtained, the fluctuation of RSS will not induce the handover. Meanwhile the network load information is needed to obtain the more optimal resource utilization, i.e. by avoiding the longer congestion. Therefore, the implementation of proposed utility for handover decision can overcome RSS fluctuation problem and frequent topology 
change in $\mathrm{V} 2 \mathrm{~V}$ communication. The results of simulations show that the proposed utility based handover method can provide the better performance than RSS-based and other relevant methods in terms of average throughput and handover rate.

\section{Acknowledgments}

The work is supported by Research Directorate of Universitas Gadjah Mada through 2019 RTA program.

\section{References}

[1] H. Hartenstein and K. P. Laberteaux, "A Tutorial Survey on Vehicular Ad Hoc Networks", IEEE Communications Magazine, Vol. 46, No. 6, pp. 164-171, 2008.

[2] IEEE, "IEEE Standard for Information technology-- Local and metropolitan area networks-- Specific requirements-- Part 11: Wireless LAN Medium Access Control (MAC) and Physical Layer (PHY) Specifications Amendment 6: Wireless Access in Vehicular Environments", IEEE Std 802.11p-2010, No. July, pp. 1-51, 2010.

[3] G. Karagiannis, O. Altintas, E. Ekici, G. Heijenk, B. Jarupan, K. Lin, and T. Weil, "Vehicular Networking: A Survey and Tutorial on Requirements, Architectures, Challenges, Standards and Solutions", IEEE Communications Surveys and Tutorials, Vol. 13, No. 4, pp. 584-616, 2011.

[4] K. Abboud, S. Member, and W. Zhuang, "Stochastic Modeling of Single-Hop Cluster Stability in Vehicular Ad Hoc Networks", IEEE Transactions on Vehicular Technology, Vol. 65, No. 1, pp. 226-240, 2016.

[5] A. Ahmed, M. Boulahia, and D. Ga, "Enabling Vertical Handover Decisions in Heterogeneous Wireless Networks: A State-of-the-Art and A Classification", IEEE Communications Surveys and Tutorials, Vol. 16, No. 2, pp. 776-811, 2014.

[6] A. Magnano, F. Xin, and A. Boukerche, "Predictive Mobile IP Handover for Vehicular Networks", In: Proc. of the 40th Annual IEEE Conference on Local Computer Networks (LCN), pp. 338-346, 2015.

[7] D. Lopes and S. Sargento, "Network Mobility for Vehicular Networks", In: Proc. of the 2014 IEEE Symposium on Computers and Communications (ISCC), pp. 1-7, 2014.

[8] R. Zagrouba, H. Hayouni, and F. Kamoun, "Handover optimization within vehicular networks", In: Proc. of the 2014 World Congress on Computer Applications and Information Systems (WCCAIS), pp. 1-5, 2014.

[9] A. Ghosh, V. V. Paranthaman, G. Mapp, and O. Gemikonakli, "Exploring efficient seamless handover in VANET systems using network dwell time", EURASIP Journal on Wireless Communications and Networking, Vol. 2014, No. 1, pp. 1-19, 2014.

[10] J. Dias, A. Cardote, F. Neves, S. Sargento, and A. Oliverira, "Seamless Horizontal and Vertical Mobility in VANET", In: Proc. of the 2012 IEEE Vehicular Networking Conference (VNC), pp. 226-233, 2012.

[11]M. Ali, H. Zoulikha, and M. Maaza, "Vertical Handover Decision Algorithm for Multimedia Streaming in VANET", Wireless Personal Communications, Vol. 95, No. 4, pp. 42814299, 2017.

[12] S. Bi, C. Chen, R. Du, and X. Guan, "Proper Handover Between VANET and Cellular Network Improves Internet Access", In: Proc. of the 2014 IEEE 80th Vehicular Technology Conference (VTC2014-Fall), pp. 1-5, 2014.

[13] K.-L. Chiu, R.-H. Hwang, and Y.-S. Chen, "A Cross Layer Fast Handover Scheme in VANET", In: Proc. of the 2009 IEEE International Conference on Communications, pp. 1-5, 2009.

[14] R. Ahmad, E. A. Sundararajan, N. E. Othman, and M. Ismail, "Handover in LTE-advanced wireless networks: state of art and survey of decision algorithm", Telecommunication Systems, Vol. 66, No. 3, pp. 533-558, 2017.

[15]A. Hasswa, N. Nasser, and H. Hassanein, "Generic Vertical Handoff Decision Function for Heterogeneous Wireless Networks", In: Proc. of Second IFIP International Conference on Wireless and Optical Communications Networks (WOCN 2005), pp. 239-243, 2005.

[16]O. Ormond and J. Murphy, "Utility-based Intelligent Network Selection in Beyond 3G Systems", In: Proc. of the 2006 IEEE International Conference on Communications, Vol. 4, pp. 1831-1836, 2006.

[17] J. M. Lee, O. S. Yang, J. K. Choi, J. I. Lim, and V. Heo, "Handover Decision Algorithm for Fixed to Mobile Handover in Heterogeneous Networks", In: Proc. of the 2006 International Conference on Wireless Communications, Networking and Mobile Computing, pp. 1-4, 2006.

[18]H. Wang, and N. Liu, "Dynamic Load Balancing and Throughput Optimization in L. T. E. Networks", In: Proc. of the 2010 5th 
International ICST Conference on Communications and Networking in China, pp. 1-5, 2010.

[19]P. Fuxjäger, A. Costantini, D. Valerio, P. Castiglione, G. Zacheo, T. Zemen, and F. Ricciato, "IEEE 802 . 11p Transmission Using GNURadio", In: Proc. of the 6th Karlsruhe Workshop on Software Radios (WSR), pp. 8386, 2010.

[20] M. Ren, L. Khoukhi, H. Labiod, J. Zhang, and $\mathrm{V}$. Vèque, "A mobility-based scheme for dynamic clustering in vehicular ad-hoc networks (VANETs)", Vehicular Communications, Vol. 9, pp. 233-241, 2017.

[21] D. Krajzewicz, J. Erdmann, M. Behrisch, and L. Bieker, "Recent Development and Applications of SUMO - Simulation of Urban MObility", International Journal on Advances in Systems and Measurements, Vol. 5, No. 3, pp. 128-138, 2012.

[22]H. Fernández, L. Rubio, V. M. RodrigoPeñarrocha, and J. Reig, "Path Loss Characterization for Vehicular Communications at $700 \mathrm{MHz}$ and $5.9 \mathrm{GHz}$ Under LOS and NLOS Conditions", IEEE Antennas and Wireless Propagation Letters, Vol. 13, pp. 931934, 2014.

[23] R. Yoneya, A. Mehbodniya, and F. Adachi, "Two Novel Handover Algorithms with Load Balancing for Heterogeneous Network", In: Proc. of the 2015 IEEE 82nd Vehicular Technology Conference (VTC2015-Fall), pp. 15, 2015. 